PROCEEDINGS OF THE

AMERICAN MATHEMATICAL SOCIETY

Volume 135, Number 10, October 2007, Pages 3151-3161

S 0002-9939(07)09019-3

Article electronically published on June 21, 2007

\title{
DIMENSION FUNCTIONS OF CANTOR SETS
}

\author{
IGNACIO GARCIA, URSULA MOLTER, AND ROBERTO SCOTTO \\ (Communicated by Michael T. Lacey)
}

\begin{abstract}
We estimate the packing measure of Cantor sets associated to nonincreasing sequences through their decay. This result, dual to one obtained by Besicovitch and Taylor, allows us to characterize the dimension functions recently found by Cabrelli et al for these sets.
\end{abstract}

\section{INTRODUCTION}

A Cantor set is a compact perfect and totally disconnected subset of the real line. In this article we consider Cantor sets of Lebesgue measure zero. Different kinds of these sets appear in many areas of mathematics, such as number theory and dynamical systems. They are also interesting in themselves as theoretical examples and counterexamples. A classical way to understand them quantitatively is through the Hausdorff measure and dimension.

A function $h:\left(0, \lambda_{h}\right] \rightarrow(0, \infty]$, where $\lambda_{h}>0$, is said to be a dimension function if it is continuous, nondecreasing and $h(x) \rightarrow 0$ as $x \rightarrow 0$. We denote by $\mathscr{D}$ the set of dimension functions.

Given $E \subset \mathbb{R}$ and $h \in \mathscr{D}$, we set $h(E)=h(|E|)$, where $|E|$ is the diameter of the set $E$.

Recall that a $\delta$-covering of a given set $E$ is a countable family of subsets of $\mathbb{R}$ covering $E$ whose diameters are less than $\delta$. The $h$-Hausdorff measure of $E$ is defined as

$$
\mathcal{H}^{h}(E)=\varliminf_{\delta \rightarrow 0}\left\{\sum_{i=1}^{\infty} h\left(U_{i}\right):\left\{U_{i}\right\} \text { is a } \delta \text {-covering of } E\right\} .
$$

We say that $E$ is an $h$-set if $0<\mathcal{H}^{h}(E)<+\infty$.

When the dimension function is $g_{s}(x)=x^{s}$, for $s \geq 0$, we set $\mathcal{H}^{s}:=\mathcal{H}^{g_{s}}\left(\mathcal{H}^{0}\right.$ is the counting measure). The Hausdorff dimension of the set $E$, denoted by $\operatorname{dim} E$, is the unique value $t$ for which $\mathcal{H}^{s}(E)=0$ if $s>t$ and $\mathcal{H}^{s}(E)=+\infty$ if $s<t$ (see Proposition 2.21). This property allows us to obtain an intuitive classification of how thin a subset of $\mathbb{R}$ of Lebesgue measure zero is.

A set $E$ is said to be dimensional if there is at least one $h \in \mathscr{D}$ that makes $E$ an $h$ set. Not all sets are dimensional (cf. [Bes39]); in fact, there are open problems about the dimensionality of certain sets, for instance, the set of Liouville numbers, which

Received by the editors May 2, 2006.

2000 Mathematics Subject Classification. Primary 28A78, 28A80.

Key words and phrases. Cantor sets, packing measure, Hausdorff dimension, dimension function.

(C)2007 American Mathematical Society Reverts to public domain 28 years from publication 
has Hausdorff dimension zero (see for example Ols03). Nevertheless, Cabrelli et al [CMMS04] showed that every Cantor set associated to a nonincreasing sequence $a$ is dimensional; that is, they constructed a function $h_{a} \in \mathscr{D}$ for which $C_{a}$ is an $h_{a}$-set. Moreover, they show that if the sequence $a$ behaves like $n^{-1 / s}$, then $h_{a} \equiv g_{s}$ (see definition below) and therefore $C_{a}$ is an $s$-set. But in other cases the behavior of these functions is not so clear. For example, there exists a sequence $a$ such that $C_{a}$ is an $\alpha$-set but $h_{a} \not \equiv g_{\alpha}$ (CHM02]). So these functions could be too general in order to give a satisfactory idea about the size of the set. To understand this situation we study the packing premeasure of these sets, which is defined as follows. A $\delta$-packing of a given set $E$ is a disjoint family of open balls centered at $E$ with diameters less than $\delta$. The h-packing premeasure of $E$ is defined as

$$
P_{0}^{h}(E)=\varlimsup_{\delta \rightarrow 0}\left\{\sum_{i=1}^{\infty} h\left(B_{i}\right):\left\{B_{i}\right\}_{i} \text { is a } \delta \text {-packing of } E\right\} .
$$

As a consequence of our main result, which is dual to the one obtained in BT54] and will be dealt with in Section 4, we are able to characterize completely when a dimension function is equivalent to a power function (Theorem 4.4). That is, for a nonincreasing sequence $a$ and $h \in \mathscr{D}$ we obtain that

$$
h \equiv g_{s} \Longleftrightarrow 0<\mathcal{H}^{s}\left(C_{a}\right) \leq P_{0}^{s}\left(C_{a}\right)<+\infty .
$$

Thus, to have that $g_{\alpha} \equiv h_{a}$, it is not only necessary that $C_{a}$ is an $\alpha$-set but also that $P_{0}^{\alpha}\left(C_{a}\right)<+\infty$.

\section{Some Remarks AND Definitions}

By the definition of $P_{0}^{h}$, it is clear that it is monotone but it is not a measure because it is not $\sigma$-additive; the $h$-packing measure $\mathcal{P}^{h}$ is obtained by a standard argument, $\mathcal{P}^{h}(E)=\inf \left\{\sum_{i=1}^{\infty} P_{0}^{h}\left(E_{i}\right): E=\bigcup_{i} E_{i}\right\}$.

As with Hausdorff measures, given a set $E$ there exists a critical value $\operatorname{dim}_{P} E$, the packing dimension of $E$, such that $\mathcal{P}^{s}(E)=0$ if $s>\operatorname{dim}_{P} E$ and $\mathcal{P}^{s}(E)=+\infty$ if $s<\operatorname{dim}_{P} E$. Analogously for the prepacking measure family $\left\{P_{0}^{s}\right\}$ we call $\Delta E$ its critical value. In Tri82 it is shown that $\Delta E$ coincides with the upper Box dimension of $E$, which we now define.

Given $0<\varepsilon<\infty$ and a nonempty bounded set $E \subset \mathbb{R}^{d}$, let $N(E, \varepsilon)$ be the smallest number of balls of radius $\varepsilon$ needed to cover $E$. The lower and upper Box dimensions of $E$ are given by

$$
\underline{\operatorname{dim}}_{B} E=\varliminf_{\varepsilon \rightarrow 0} \frac{\log N(E, \varepsilon)}{\log 1 / \varepsilon} \quad \text { and } \quad \varlimsup_{\operatorname{dim}_{B}} E=\varlimsup_{\varepsilon \rightarrow 0} \frac{\log N(E, \varepsilon)}{\log 1 / \varepsilon}
$$

respectively. When the lower and upper limits coincide, the common value is the Box dimension of $E$ and we denote it by $\operatorname{dim}_{B} E$.

Note that $\mathcal{H}^{h}(E) \leq \mathcal{P}^{h}(E)$ when $h$ is a doubling function (TT85]). Moreover, $\operatorname{dim} E \leq \operatorname{dim}_{P} E \leq \Delta E \leq 1$ and $\operatorname{dim} E \leq \underline{\operatorname{dim}}_{B} E \leq \overline{\operatorname{dim}}_{B} E$; all these inequalities can be strict (Tri82).

We observe that $\mathcal{H}^{h}$ is Borel-regular, which is also true if we only require the right continuity of $h \in \mathscr{D}$ Rog98. On the other hand, $\mathcal{P}^{h}$ and $P_{0}^{h}$ are also Borelregular, but in this case one has to require that $h \in \mathscr{D}$ be left continuous [TT85, Lemma 3.2). Since in this paper we are concerned with all of these measures, we require that $h \in \mathscr{D}$ should be continuous. 
Now we define a partial order in $\mathscr{D}$, which will be our way of comparing the elements of $\mathscr{D}$.

Definition 2.1. Let $f$ and $h$ be in $\mathscr{D}$.

- $f$ is smaller than $h$, denoted by $f \prec h$, if

$$
\lim _{x \rightarrow 0} h(x) / f(x)=0 \text {. }
$$

- $f$ is equivalent to $h$, denoted by $f \equiv h$, if

$$
0<c_{1}=\varliminf_{x \rightarrow 0} \frac{h(x)}{f(x)} \leq \varlimsup_{x \rightarrow 0} \frac{h(x)}{f(x)}=c_{2}<+\infty .
$$

We set $f \preceq g$ when $f \prec g$ or $f=g$. We say that $f$ and $g$ are not comparable if none of the relations $f \prec g, g \prec f$ or $f \equiv g$ holds.

Notice that this definition is consistent with the usual law of the power functions: $x^{s} \prec x^{t}$ iff $s<t$.

Proposition 2.2. If $\nu^{f}$ is $\mathcal{H}^{f}$ or $P_{0}^{f}$ or $\mathcal{P}^{f}$, then we have the following.

i) If $f \prec h$, then: $\nu^{f}(E)<\infty \Longrightarrow \nu^{h}(E)=0$.

ii) If $f \equiv h$, then:

a) $\nu^{f}(E)<\infty \Longleftrightarrow \nu^{h}(E)<\infty$;

b) $0<\nu^{f}(E) \Longleftrightarrow 0<\nu^{h}(E)$;

c) in particular, $E$ is an $f$-set if and only if $E$ is an h-set.

The proof of this proposition for the Hausdorff measure case can be found in Rog98; the packing cases are analogous.

\section{Cantor sets associated to nOnincreasing SEQUences}

Definition 3.1. Let $a=\left\{a_{k}\right\}$ be a positive, nonincreasing and summable sequence. Let $I_{a}$ be a closed interval of length $\sum_{k=1}^{\infty} a_{k}$. We define $\mathscr{C}_{a}$ to be the family of all closed sets $E$ contained in $I_{a}$ that are of the form $E=I_{a} \backslash \bigcup_{j \geq 1} U_{j}$, where $\left\{U_{j}\right\}$ is a disjoint family of open intervals contained in $I_{a}$ such that $\left|\bar{U}_{k}\right|=a_{k} \forall k$.

With this definition, every element of $\mathscr{C}_{a}$ has Lebesgue measure zero. From this family, we consider the Cantor set $C_{a}$ associated to the sequence $a$ constructed as follows: In the first step, we remove from $I_{a}$ an open interval of length $a_{1}$, resulting in two closed intervals $I_{1}^{1}$ and $I_{2}^{1}$. Having constructed the $k$-th step, we get the closed intervals $I_{1}^{k}, \ldots, I_{2^{k}}^{k}$ contained in $I_{a}$. The next step consists in removing from $I_{j}^{k}$ an open interval of length $a_{2^{k}+j-1}$, obtaining the closed intervals $I_{2 j-1}^{k+1}$ and $I_{2 j}^{k+1}$. Then we define $C_{a}:=\bigcap_{k=1}^{\infty} \bigcup_{j=1}^{2^{k}} I_{j}^{k}$. Note that in this construction there is a unique form of removing open intervals at each step; also, note that for this construction it is not necessary for the sequence to be nonincreasing.

Remark 3.2. Since $a$ is nonincreasing, the sequence $\left\{\left|I_{j}^{k}\right|\right\}_{(k, j)}$, with $1 \leq j \leq 2^{k}$ and $k \geq 1$, is (lexicographically) nonincreasing.

We associate to the sequence $a$ the summable sequences $\underline{a}$ and $\bar{a}$ defined as

$$
\underline{a}_{n}=a_{2^{r+1}-1} \text { and } \bar{a}_{n}=a_{2^{r}}
$$

with $2^{r} \leq n<2^{r+1}$; thus $\underline{a}_{n} \leq a_{n} \leq \bar{a}_{n} \forall n$. The sets $C_{\underline{a}}$ and $C_{\bar{a}}$ are uniform, which means that for each $k \geq 1$, the closed intervals of the $k$-th step have all the 
same length. Observe that for each uniform Cantor set the Hausdorff and lower box dimensions coincide (cf. [CHM97]).

To study the Hausdorff measure and dimension of these sets, Besicovitch and Taylor in [BT54] studied the decay of the sequence $b_{n}=r_{n} / n$, where $r_{n}=\sum_{j \geq n} a_{j}$. They introduced the number

$$
\alpha(a)=\varliminf_{n \rightarrow \infty} \alpha_{n}, \text { where } n b_{n}^{\alpha_{n}}=1 \forall n,
$$

and showed that $\operatorname{dim} E \leq \alpha(a)$ for all $E \in \mathscr{C}_{a}$. In fact, $\operatorname{dim} C_{a}=\alpha(a)$ (see, for example, CMMS04). Also, as a consequence of another result in [BT54] (see Proposition 4.1 below),

$$
\operatorname{dim} C_{a}=\inf \left\{s>0: \underline{\left.\lim n b_{n}^{s}<+\infty\right\} .}\right.
$$

In this paper (Theorem 4.2) we obtain the symmetrical result for the packing case:

$$
\Delta C_{a}=\inf \left\{s>0: \varlimsup \lim n b_{n}^{s}<+\infty\right\} .
$$

On the other hand, the box dimensions of the sets in $\mathscr{C}_{a}$ are related to the constants

$$
\gamma(a)=\varliminf_{k \rightarrow \infty} \frac{\log 1 / k}{\log a_{k}} \text { and } \beta(a)=\varlimsup_{k \rightarrow \infty} \frac{\log 1 / k}{\log a_{k}} .
$$

In fact, by Propositions 3.6 and 3.7 of Falconer's book [Fal97, the box dimension of $E \in \mathscr{C}_{a}$ exists if and only if $\gamma(a)=\beta(a)$, and in this case $\operatorname{dim}_{B} E=\beta(a)$. Moreover, as Falconer remarks, every $E \in \mathscr{C}_{a}$ has the same upper box dimension and the same lower box dimension. The former equals $\beta(a)$, which in Tri95] is shown to be equal to $\varlimsup_{n \rightarrow \infty} \alpha_{n}$. In the following proposition we obtain the symmetrical result, that is, the latter is $\underline{\lim }_{n \rightarrow \infty} \alpha_{n}$, but observe that $\gamma(a) \leq \underline{\operatorname{dim}}_{B} C_{a}$ and the inequality can be strict (cf. Propositions 3 and 5 in [CMMS04]).

Proposition 3.3. For every nonincreasing sequence a we have that $\underline{\operatorname{dim}}_{B} C_{a}=$ $\operatorname{dim} C_{a}=\alpha(a)$.

Proof. First note that $\underline{\lim } n b_{n}^{s} \sim \underline{\lim } n \bar{b}_{n}^{s}$. In fact, to see the nontrivial inequality, if $\left\{n_{j}\right\}$ is a subsequence of the natural numbers let $2^{l_{j}-1} \leq n_{j}<2^{l_{j}}$, so $2^{l_{j}} b_{2^{l_{j}}}^{s} \leq$

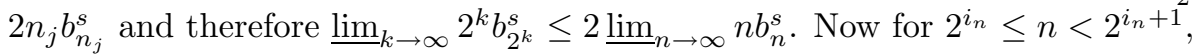

$$
\begin{aligned}
\bar{r}_{n} & \leq \sum_{k \geq 2^{i_{n}}} \bar{a}_{k}=\sum_{k=0}^{\infty} 2^{i_{n}+k} a_{2^{i_{n}+k}} \\
& \leq 2 \sum_{k=0}^{\infty} 2^{i_{n}+k-1} a_{2^{i_{n}+k}-1}=2 \underline{r}_{2^{i_{n}-1}} \leq 2 r_{2^{i_{n}-1}} ;
\end{aligned}
$$

hence $\underline{\lim }_{n \rightarrow \infty} n \bar{b}_{n}^{s} \leq 4 \underline{\lim }_{j \rightarrow \infty} 2^{j} b_{2^{j}}^{s} \leq 8 \underline{\lim }_{n \rightarrow \infty} n b_{n}^{s}$.

Then by (3.1) $\operatorname{dim} C_{a}=\operatorname{dim} C_{\bar{a}}$, and by Proposition 3.1 of [CHM97] we have that $\operatorname{dim} C_{a}=\underline{\operatorname{dim}}_{B} C_{\bar{a}}$. On the other hand, $N\left(\varepsilon, C_{a}\right) \leq N\left(\varepsilon, C_{\bar{a}}\right)\left(C_{a}\right.$ can be mapped to $C_{\bar{a}}$ by a bijection which preserves the order; then, if two points of $C_{\bar{a}}$ are contained in an open set $U$, the corresponding points of $C_{a}$ will be contained in an open set of the same diameter as $U$ ), so $\underline{\operatorname{dim}}_{B} C_{a} \leq \underline{\operatorname{dim}}_{B} C_{\bar{a}}$.

This proposition in particular asserts that $\underline{\operatorname{dim}}_{B} E=\inf \left\{s>0: \underline{\lim } n b_{n}^{s}<+\infty\right\}$ for any $E \in \mathscr{C}_{a}$. On the other hand, since the critical exponent associated to 
the packing premeasure of a given set is the upper box dimension, it is clear that $\overline{\operatorname{dim}}_{B} E=\inf \left\{s>0: \varlimsup \lim n b_{n}^{s}<+\infty\right\}$.

\section{MAin Results}

The next proposition is a result that generalizes the one established in BT54 for the functions $g_{s}$ to any function $h \in \mathscr{D}$ (cf. CHM02]). It shows that $\mathcal{H}^{h}\left(C_{a}\right)$ behaves like $n h\left(b_{n}\right)$ when $n \rightarrow \infty$.

Proposition 4.1. For $h \in \mathscr{D}, \frac{1}{4} \underline{\lim }_{n \rightarrow \infty} n h\left(b_{n}\right) \leq \mathcal{H}^{h}\left(C_{a}\right) \leq 4 \underline{\lim }_{n \rightarrow \infty} n h\left(b_{n}\right)$.

Proof. The lower inequality $\frac{1}{4} \varliminf_{n \rightarrow \infty} n h\left(b_{n}\right) \leq \mathcal{H}^{h}\left(C_{a}\right)$ is obtained in exactly the same way as in BT54 replacing $g_{s}$ by $h$. Hence, we only show here that $\mathcal{H}^{h}\left(C_{a}\right) \leq 4 \underline{\lim } n h\left(b_{n}\right)$. We begin by noting that $\left|\underline{I}_{j}^{k}\right|=\sum_{i=0}^{\infty} 2^{i} \underline{a}_{2^{k+i}}=\underline{b}_{2^{k}}$ for $1 \leq j \leq 2^{k}, k>0$. Then, from the identities

$$
\begin{aligned}
\left|I_{1}^{k}\right| & =a_{2^{k}}+\left(a_{2^{k+1}}+a_{2^{k+1}+1}\right)+\left(a_{2^{k+2}}+a_{2^{k+2}+1}+a_{2^{k+2}+2}+a_{2^{k+2}+3}\right)+\ldots, \\
\left|\underline{I}_{1}^{k-1}\right| & =a_{2^{k}-1}+\left(a_{2^{k+1}-1}+a_{2^{k+1}-1}\right)+\left(a_{2^{k+2}-1}+a_{2^{k+2}-1}+a_{2^{k+2}-1}+a_{2^{k+2}-1}\right)+\ldots
\end{aligned}
$$

and Remark 3.2, we have the following estimate:

$$
\left|I_{j}^{k}\right| \leq\left|I_{1}^{k}\right| \leq\left|\underline{I}_{1}^{k-1}\right|=\underline{b}_{2^{k-1}} \leq b_{2^{k-1}}
$$

and hence $\sum_{j=1}^{2^{k}} h\left(I_{j}^{k}\right) \leq 2^{k} h\left(b_{2^{k-1}}\right)$. Given $\delta>0$ there exists $k_{\delta}$ such that $\left|I_{1}^{k}\right|<\delta$ for $k \geq k_{\delta}$; that is, for $k \geq k_{\delta}$, the closed intervals of the $k$-th step form a $\delta$-covering of $C_{a}$, which implies $\mathcal{H}^{h}\left(C_{a}\right) \leq 2 \underline{\lim }_{k \rightarrow \infty} 2^{k} h\left(b_{2^{k}}\right)$, and therefore $\mathcal{H}^{h}\left(C_{a}\right) \leq 4 \underline{\lim } n h\left(b_{n}\right)$.

Our main result is the following theorem, which is in some sense dual to the previous one.

Theorem 4.2. For any $h \in \mathscr{D}, \frac{1}{8} \varlimsup_{n \rightarrow \infty} n h\left(b_{n}\right) \leq P_{0}^{h}\left(C_{a}\right) \leq 8 \varlimsup_{n \rightarrow \infty} n h\left(b_{n}\right)$.

Proof. For the first inequality, suppose that $\overline{\lim }_{n \rightarrow \infty} n h\left(b_{n}\right)>d$. To prove that $P_{0}^{h}\left(C_{a}\right) \geq d / 8$, for each $\delta>0$ it suffices to find a $\delta$-packing $\left\{B_{i}\right\}_{i}$ of $C_{a}$ with $\sum_{i} h\left(B_{i}\right)>d / 8$. Observe that, since $\left\{a_{n}\right\}$ is nonincreasing, then

$$
h\left(b_{2^{k}}\right)=h\left(2^{-k} \sum_{1 \leq i \leq 2^{k}}\left|I_{i}^{k}\right|\right) \leq h\left(I_{1}^{k}\right) .
$$

By hypothesis there exists a subsequence $\left\{n_{j}\right\}_{j \geq 1}$ such that $n_{j} h\left(b_{n_{j}}\right)>d$. For each $j$, let $k_{j}$ be the unique integer for which $2^{k_{j}} \leq n_{j}<2^{k_{j}+1}$; since $\left\{b_{n}\right\}_{n}$ is decreasing, it follows from (4.2) that

$$
d<n_{j} h\left(b_{n_{j}}\right)<2^{k_{j}+1} h\left(b_{2^{k_{j}}}\right) \leq 2^{k_{j}+1} h\left(I_{1}^{k_{j}}\right) .
$$

Pick $j$ large enough so that $\left|I_{1}^{k_{j}}\right|<\delta$; since the diameter of this interval is smaller than the diameter of every interval of the $k_{j}-1$ step, the family of intervals $\left\{B_{i}\right\}_{i=1}^{2^{k_{j}-2}}$, where $B_{i}$ is centered at the right endpoint of the interval $I_{2 i-1}^{k_{j}-1}$ and $\left|B_{i}\right|=$ $\left|I_{1}^{k_{j}}\right|$, turns out to be a $\delta$-packing of $C_{a}$, and by (4.3), $\sum_{i} h\left(B_{i}\right)=2^{k_{j}-2} h\left(I_{1}^{k_{j}}\right)>$ $d / 8$.

For the second inequality, if $\left\{B_{i}\right\}_{i=1}^{N}$ is a $\delta$-packing of $C_{a}$, we define

$$
k_{i}=\min \left\{k: I_{j}^{k} \subset B_{i} \text { for some } 1 \leq j \leq 2^{k}\right\} .
$$


By the definition of $k_{i}, B_{i}$ is centered at a point of an interval of the $k_{i}-1$ step, but it does not contain the interval, so $\left|B_{i}\right|<\left|I_{j_{i}}^{k_{i}-2}\right|$, where $I_{j_{i}}^{k_{i}-2}$ is the interval of the $k_{i}-2$ step which contains the center of $B_{i}$. Then, by the monotony of $h$ and (4.1),

$$
\sum_{i=1}^{N} h\left(B_{i}\right) \leq \sum_{i=1}^{N} h\left(I_{j_{i}}^{k_{i}-2}\right) \leq \sum_{i=1}^{N} h\left(b_{2^{k_{i}-3}}\right) .
$$

Furthermore, we can assume that $\left|B_{1}\right| \geq \ldots \geq\left|B_{N}\right|$, so $k_{1} \geq \ldots \geq k_{N}$. Let $l_{1}>\ldots>l_{M}$ denote those $k_{i}$ 's that do not repeat themselves. Let $\theta_{m}$ be the number of repeated $l_{m}$ 's; i.e., $\theta_{m}$ tells us how many of the $B_{i}$ 's contain an interval of the $l_{m}$-th step but none of the previous ones. Since $\left\{B_{i}\right\}_{i=1}^{N}$ is a disjoint family, $\theta_{1}$ cannot be greater than the number of intervals of step $l_{1}$, which is $2^{l_{1}}$; each ball of the packing associated to $l_{1}$ contains $2^{l_{2}-l_{1}}$ intervals of step $l_{2}$ and therefore $\theta_{2} \leq 2^{l_{2}}-\theta_{1} 2^{l_{2}-l_{1}}$. Continuing with this process we obtain

$$
\theta_{M} \leq 2^{l_{M}}-\sum_{i=1}^{M-1} \theta_{i} 2^{l_{M}-l_{i}}=2^{l_{M}}\left(1-\sum_{i=1}^{M-1} \frac{\theta_{i}}{2^{l_{i}}}\right)
$$

and hence $\sum_{i=1}^{M} \theta_{i} / 2^{l_{i}} \leq 1$.

Finally, choose $\delta$ sufficiently small such that $2^{l_{1}-3} \geq n_{0}$, where

$$
\sup _{n \geq n_{0}} n h\left(b_{n}\right) \leq \varlimsup_{n \rightarrow \infty} n h\left(b_{n}\right)+\varepsilon .
$$

Then,

$$
\sum_{i=1}^{N} h\left(B_{i}\right) \leq \sum_{j=1}^{M} \frac{\theta_{j}}{2^{l_{j}-3}} 2^{l_{j}-3} h\left(b_{2^{l_{j}-3}}\right) \leq 8\left(\varlimsup_{n \rightarrow \infty} n h\left(b_{n}\right)+\varepsilon\right),
$$

and from this the theorem follows.

We are now ready to complete the characterization promised in the introduction.

Note that the function $h_{a} \in \mathscr{D}$ found in CMMS04 is defined in such a way that $h_{a}\left(b_{n}\right)=1 / n$ for all $n$. Then, by Theorem 4.2 we obtain that $P_{0}^{h_{a}}\left(C_{a}\right)<+\infty$, and therefore the Cantor sets associated to nonincreasing sequences not only are dimensional but also have a dimension function which simultaneously regularizes the covering and packing processes in the construction of the measures. We need the following lemma.

Lemma 4.3. Let $h, g \in \mathscr{D}$. In addition, assume that $0<\mathcal{H}^{h}\left(C_{a}\right) \leq P_{0}^{h}\left(C_{a}\right)<$ $+\infty$. Then

a) $h \equiv g \Longleftrightarrow 0<\underline{\lim }_{n \rightarrow \infty} n g\left(b_{n}\right) \leq \varlimsup_{n \rightarrow \infty} n g\left(b_{n}\right)<+\infty$;

b) $g \prec h \Longleftrightarrow \lim _{n \rightarrow \infty} n g\left(b_{n}\right)=+\infty$;

c) $h \prec g \Longleftrightarrow \lim _{n \rightarrow \infty} n g\left(b_{n}\right)=0$.

Proof. By Proposition 4.1 and Theorem 4.2, since $\mathcal{H}^{h}\left(C_{a}\right)>0$ and $P_{0}^{h}\left(C_{a}\right)<+\infty$, there are constants $0<c_{h}$ and $C_{h}<+\infty$ such that $c_{h} 1 / n \leq h\left(b_{n}\right) \leq C_{h} 1 / n$, and all three necessary conditions follow. On the other hand, if $\left\{y_{j}\right\}$ is a sequence that decreases to 0 , then there exists a subsequence $\left\{n_{j}\right\}$ such that $b_{n_{j}+1} \leq y_{j}<b_{n_{j}}$. Therefore

$$
\frac{g\left(y_{j}\right)}{h\left(y_{j}\right)} \leq \frac{g\left(b_{n_{j}}\right)}{h\left(b_{n_{j}+1}\right)} \leq 2 c_{h}^{-1} n_{j} g\left(b_{n_{j}}\right)
$$


and

$$
\frac{g\left(y_{j}\right)}{h\left(y_{j}\right)} \geq\left(2 C_{h}\right)^{-1}\left(n_{j}+1\right) g\left(b_{n_{j}+1}\right),
$$

from which the sufficient conditions also follow.

We have now the main theorem of this part.

Theorem 4.4. Let a be a nonincreasing sequence, and let $h \in \mathscr{D}$ be such that $0<\mathcal{H}^{h}\left(C_{a}\right) \leq P^{h}\left(C_{a}\right)<+\infty$. Then, for $g \in \mathscr{D}$ we have:

a) $h \equiv g \Longleftrightarrow 0<\mathcal{H}^{g}\left(C_{a}\right) \leq P_{0}^{g}\left(C_{a}\right)<+\infty$;

b) $g \prec h \Longleftrightarrow \mathcal{H}^{g}\left(C_{a}\right)=+\infty$;

c) $h \prec g \Longleftrightarrow P_{0}^{g}\left(C_{a}\right)=0$.

In particular, $h$ will be equivalent to $x^{s}$ if and only if $0<\mathcal{H}^{s}\left(C_{a}\right) \leq P_{0}^{s}\left(C_{a}\right)<+\infty$.

Proof. The proof is immediate from Proposition 4.1. Theorem 4.2 and Lemma 4.3 .

Corollary 4.5. Let $\alpha=\operatorname{dim} C_{a}$ and $\beta=\Delta C_{a}$. Take $h_{a}$ to be the dimension function of $C_{a}$.

a) If $s<\alpha$ and $\beta<t$, then $g_{s} \prec h_{a}$ and $h_{a} \prec g_{t}$. If $\mathcal{H}^{\alpha}\left(C_{a}\right)=+\infty$, then $g_{\alpha} \prec h_{a}$, and if $\mathcal{H}^{\alpha}\left(C_{a}\right)<+\infty$, then $g_{\alpha} \nprec h_{a}$. If $P_{0}^{\beta}\left(C_{a}\right)=0$, then $h_{a} \prec g_{\beta}$, and if $P_{0}^{\beta}\left(C_{a}\right)>0$, then $h_{a} \nprec g_{\beta}$.

b) In the case $\alpha<\beta, h_{a} \not \equiv g_{s}$ for no $s \geq 0$. Moreover, if $\alpha<t<\beta$, then $h_{a}$ and $g_{t}$ are not comparable. In the limit cases, if $\mathcal{H}^{\alpha}\left(C_{a}\right)<+\infty$, then $h_{a}$ and $g_{\alpha}$ are not comparable, and if $P_{0}^{\beta}\left(C_{a}\right)>0, h_{a}$ and $g_{\beta}$ are not comparable.

Proof. In the case $\mathcal{H}^{\alpha}\left(C_{a}\right)<+\infty$, if $g_{a}$ were to satisfy $g_{\alpha} \prec h_{a}$, Proposition 2.2 would imply that $\mathcal{H}^{h_{a}}\left(C_{a}\right)=0$, which is a contradiction. Hence $g_{\alpha} \nprec h_{a}$. Analogously, $P_{0}^{\beta}\left(C_{a}\right)>0$ implies that $h_{a} \nprec g_{\beta}$, for if not $P_{0}^{h_{a}}\left(C_{a}\right)=+\infty$, contradicting Theorem 4.2. The rest of the claims of item $a$ ) are immediate from Theorem 4.4.

To show $b$ ), if $h_{a} \equiv g_{s}$ for some $s \geq 0$, then $0<\mathcal{H}^{s}\left(C_{a}\right) \leq P_{0}^{s}\left(C_{a}\right)<+\infty$; therefore $\alpha=\beta$. Moreover, by Proposition 2.2, it follows that $g_{s} \nprec h$ when $s>$ $\operatorname{dim} C_{a}$, and also $h_{a} \nprec g_{s}$ if $0 \leq s<\beta$.

Note that, since $\alpha=\underline{\operatorname{dim}}_{B} C_{a}$ and $\beta=\overline{\operatorname{dim}}_{B} C_{a}$, part $b$ ) of this corollary emphasizes that in order to have $h_{a} \equiv g_{s}$ we need $\underline{\operatorname{dim}}_{B} C_{a}=\overline{\operatorname{dim}}_{B} C_{a}$. But this latter condition and the fact that $C_{a}$ is an $\alpha$-set are not sufficient to ensure the equivalence, and thus the hypothesis of Theorem $4.4 \mathrm{a}$ ) cannot be weakened to existence of box dimension and $C_{a}$ being an $\alpha$-set.

Example 4.6. For each $0<s<1$ there exists a Cantor set $C_{a}$ associated to a nonincreasing sequence for which $\operatorname{dim} C_{a}=\overline{\operatorname{dim}}_{B} C_{a}=s$ and $0<\mathcal{H}^{s}\left(C_{a}\right)<+\infty$, but $P_{0}^{s}\left(C_{a}\right)=+\infty$.

To construct this sequence we set $\lambda_{k}=\left(\frac{1}{2}\right)^{\frac{k}{s+\varepsilon_{k}}}$, where

$$
\varepsilon_{k}=\left\{\begin{array}{cc}
\frac{s \log l}{k}, & 2^{m}<k \leq 2^{m+1}, l=k-2^{m}, m \text { even, } \\
0, & \text { otherwise. }
\end{array}\right.
$$

Let us define $a_{j}=\lambda_{k}$ for $2^{k-1} \leq j<2^{k}$ and $k \geq 1$. The uniform Cantor set $C_{a}$ gives us the example. In fact, it is easy to check that $a$ is summable, decreasing 
and, using (3.3), that $\operatorname{dim} C_{a}=\overline{\operatorname{dim}}_{B} C_{a}=s$. Next we check the claims about the measures:

a) $0<\underline{\lim }_{n \rightarrow \infty} n b_{n}^{s}$ : Since $b_{2^{k}}=\sum_{i \geq 0} 2^{i} \lambda_{k+1+i}$ and $\lambda_{j} \geq\left(\frac{1}{2}\right)^{\frac{j}{s}} \quad \forall j$, we have that

$$
2^{k} b_{2^{k}}^{s} \geq 2^{k}\left(\sum_{i \geq 0}\left(\frac{1}{2}\right)^{\frac{k+1+i}{s}-i}\right)^{s}=\frac{1}{2}\left(\sum_{i \geq 0}\left(\frac{1}{2}\right)^{\left(\frac{1}{s}-1\right) i}\right)^{s}=c_{s}>0
$$

and remember that $\varliminf_{n \rightarrow \infty} n b_{n}^{s} \sim \varliminf_{k \rightarrow \infty} 2^{k} b_{2^{k}}^{s}$.

b) $\varlimsup_{n \rightarrow \infty} n b_{n}^{s}=+\infty$ : Let $n_{j}=2^{2^{j}-1}, j$ odd. Then $b_{n_{j}}=\sum_{i \geq 0} 2^{i} \lambda_{2^{j}+i}>$ $\lambda_{2^{j}} ;$ therefore,

$$
n_{j} b_{n_{j}}^{s} \geq 2^{2^{j}-1} \lambda_{2^{j}}^{s}=2^{\frac{2^{j}(j-1) \log 2}{2^{j}+(j-1) \log 2}-1}
$$

which increases to infinity with $j$.

c) $\underline{\lim }_{n \rightarrow \infty} n b_{n}^{s}<+\infty$ : Now we set $n_{j}=2^{2^{j}}$ for $j$ odd and observe that

$$
n_{j} b_{n_{j}}^{s}=n_{j}\left(\sum_{i \geq 0} 2^{i} \lambda_{2^{j}+1+i}\right)^{s}=\left(\sum_{i \geq j} \sum_{l=1}^{2^{i}} 2^{2^{i}+l+2^{j}\left(\frac{1-s}{s}\right)-1} \lambda_{2^{i}+l}\right)^{s} .
$$

Each sum in $l$ is bounded by a geometric term; more precisely, if $j$ is sufficiently large there is a constant $C_{s}$ depending only on $s$ such that

$$
\sum_{l=1}^{2^{i}} 2^{2^{i}+l+2^{j}\left(\frac{1-s}{s}\right)} \lambda_{2^{i}+l} \leq C_{s}\left(\frac{1}{2}\right)^{i-j}
$$

or equivalently,

$$
\sum_{l=1}^{2^{i}} 2^{l} \lambda_{2^{i}+l} \leq C_{s}\left(\frac{1}{2}\right)^{2^{i}+2^{j}\left(\frac{1-s}{s}\right)+i-j} .
$$

This is easy to check when $i$ is odd. For $i$ even we obtain $\sum_{l=1}^{2^{i}} 2^{l} \lambda_{2^{i}+l}<$ $C_{s}\left(\frac{1}{2}\right)^{\frac{2^{i}}{s+\varepsilon}}$ for small $\varepsilon$. Thus (4.6) will hold if

$$
2^{i}\left(\frac{1-(s+\varepsilon)}{s+\varepsilon}\right) \geq 2^{j}\left(\frac{1-s}{s}\right)+i-j .
$$

But notice that (4.7) is true for all $i>j$ choosing $\varepsilon$ sufficiently small and $j$ large enough so that $\varepsilon_{2^{j}}<\varepsilon$.

Remark 4.7. Proposition 4.1 and Theorem 4.2 are not valid in general as the following arguments show.

If $a$ is a nonincreasing sequence and $\tilde{a}$ is any rearrangement of $a$, then $r_{n}^{a} \leq r_{n}^{\tilde{a}}$; hence, by (3.1) and since $C_{\tilde{a}} \in \mathscr{C}_{a}$,

$$
\inf \left\{s>0: \underline{\lim } n\left(b_{n}^{\tilde{a}}\right)^{s}<+\infty\right\} \geq \operatorname{dim} C_{a} \geq \operatorname{dim} C_{\tilde{a}}
$$

and each positive and summable sequence $a$ has a rearrangement $z$ for which $\operatorname{dim} C_{z}=0$ (cf. CMPS05]). This shows the counterexample for Proposition 4.1 . 
In the case of Theorem 4.2 let us see that (3.2) need not be true for a general sequence. Let $\tilde{a}$ be any rearrangement of $a$. Let $\eta(\tilde{a}):=\inf \left\{s>0: \overline{\lim } n\left(b_{n}^{\tilde{a}}\right)^{s}<\right.$ $+\infty\}$ and consider $\beta(\tilde{a})$ as defined in Section 3. Note that if $t>\eta(\tilde{a})$, then $\tilde{a}_{n}<$ $C n^{1-1 / t} \forall n$, and hence $\beta(\tilde{a}) \leq \frac{t}{1-t}$, which implies that $\beta(\tilde{a}) \leq \frac{\eta(\tilde{a})}{1-\eta(\tilde{a})}$. Therefore $\frac{\beta(\tilde{a})}{1+\beta(\tilde{a})} \leq \eta(\tilde{a})$. Thus, to show that this theorem fails in general, we exhibit a nonincreasing sequence $a$ and a rearrangement $\tilde{a}$ of it for which

$$
\beta(a)<\frac{\beta(\tilde{a})}{1+\beta(\tilde{a})},
$$

and therefore $\Delta C_{\tilde{a}}=\Delta C_{a}=\beta(a)<\eta(\tilde{a})$, where $\Delta C_{\tilde{a}}$ is the critical exponent for $P_{0}^{s}$, the packing premeasure. For $a_{n}=\left(\frac{1}{n}\right)^{p}$, we set

$$
\tilde{a}_{n}=\left\{\begin{array}{cc}
a_{3^{k}}, & n=\left\lceil\log 3^{k}\right\rceil, n \neq 3^{j} \forall j, \\
a_{\left\lceil\log 3^{k}\right\rceil}, & n=3^{k}, n \neq\left\lceil\log 3^{j}\right\rceil \forall j \\
a_{n}, & \text { otherwise, }
\end{array}\right.
$$

where $\lceil s\rceil$ denotes the smallest integer greater than $s$. Observe that this is a rearrangement of $a$ since the sequence $\left\{\left\lceil\log 3^{k}\right\rceil\right\}$ is strictly increasing and $\left\lceil\log 3^{3^{l}}\right\rceil \neq$ $3^{j}$ for each $l$ and each $j$. Then it is easy to check that $\beta(a)=1 / p$ and that $\beta(\tilde{a})=\frac{\log 3}{p}$. Therefore (4.8) holds for $p>\frac{\log 3}{\log 3-1}$.

However these propositions keep on being true if we ask the Cantor set to be uniform with no further assumptions on the sequence. This can be seen in Theorem 4.2 since inequalities (4.3) and (4.4), where the decay assumption is needed, are still true for uniform Cantor sets. For Proposition 4.1] note that Lemma 4 of [BT54] does not need the decay. Moreover, to each Cantor set associated to a nonincreasing sequence corresponds a uniform Cantor set with equivalent $h$-Hausdorff measure and $h$-packing premeasure.

Finally we give an application of the results of this section.

Let $p>1$ and $q \in \mathbb{R}$. Consider the sequence $a$ defined by $a_{n}=(\log n)^{q} / n^{p}$, $\forall n>1$. We denote by $C_{p, q}$ the Cantor set associated to $a$ and define the dimension function $h_{p, q}(x)=x^{\frac{1}{p}} /(-\log x)^{\frac{q}{p}}$.

Since $\gamma(a)=\beta(a)=1 / p$, we have that $\operatorname{dim} C_{p, q}=\operatorname{dim}_{B} C_{p, q}=1 / p$ for any $q$. Moreover, if $q=0, C_{p}=C_{p, 0}$ is a $1 / p$-set (cf. CMPS05]). Even more, as in this case $r_{n} \sim 1 / n^{p-1}$, Theorem 4.2 implies that $P_{0}^{\frac{1}{p}}\left(C_{a}\right)<+\infty$. The next corollary extends these results.

Corollary 4.8. With the above notation, $0<\mathcal{H}^{h_{p, q}}\left(C_{p, q}\right) \leq P_{0}^{h_{p, q}}\left(C_{p, q}\right)<+\infty$. In particular, $C_{p, q}$ is an $h_{p, q}$-set.

Proof. We show that $r_{n} \sim(\log n)^{q} / n^{p-1}$ and from this it is easy to see that $h_{p, q}\left(b_{n}\right) \sim 1 / n$. First suppose that $q \geq 0$. In this case we have that

$$
r_{n} \geq(\log n)^{q} \sum_{k \geq n} k^{-p} \geq c_{1} \frac{(\log n)^{q}}{n^{p-1}}
$$

and

$$
r_{n} \leq \int_{n-1}^{\infty} \frac{(\log t)^{q}}{t^{p}} d t
$$


so integrating by parts we get

$$
\begin{aligned}
r_{n} & \leq \frac{1}{p-1}\left(\frac{(\log (n-1))^{q}}{(n-1)^{p-1}}+q \int_{n-1}^{\infty} \frac{(\log t)^{q-1}}{t^{p}} d t\right) \\
& =c_{p} \frac{(\log n)^{q}}{n^{p-1}}+O\left(\frac{(\log n)^{q-1}}{n^{p-1}}\right) \\
& \leq c_{2} \frac{(\log n)^{q}}{n^{p-1}},
\end{aligned}
$$

where $c_{1}$ and $c_{2}$ are constants depending only on $p$ and $q$.

Now suppose that $q<0$. In this case it is easy to see that $r_{n} \leq c_{3}(\log n)^{q} / n^{p-1}$. On the other hand, integrating by parts twice and since $q(q-1)>0$ we obtain

$$
\begin{aligned}
r_{n} & \geq \frac{1}{p-1}\left(\frac{(\log n)^{q}}{n^{p-1}}+\frac{q}{p-1} \frac{(\log n)^{q-1}}{n^{p-1}}+\frac{q(q-1)}{p-1} \int_{n}^{\infty} \frac{(\log t)^{q-2}}{t^{p-1}} d t\right) \\
& \geq \frac{1}{p-1} \frac{(\log n)^{q}}{n^{p-1}}\left(1+\frac{q}{p-1}(\log n)^{-1}\right) ;
\end{aligned}
$$

taking $n$ sufficiently large it follows that $r_{n} \geq c_{4}(\log n)^{q} / n^{p-1}$.

Note that $h_{p, q} \preceq h_{s, t}$ if and only if $(1 / p, q) \leq_{l}(1 / s, t)\left(\leq_{l}\right.$ denote the lexicographical order in $(0,1) \times \mathbb{R})$. Define $\mathcal{H}^{\frac{1}{p}, q}=\mathcal{H}^{h_{p, q}}$, so that $\mathcal{H}^{\frac{1}{p}, 0}=\mathcal{H}^{\frac{1}{p}}$. As a consequence of the above result we can conclude the following. If $q<0,(1 / p, q)<_{l}(1 / p, 0)$, and since $\mathcal{H}^{p, q}\left(C_{p, q}\right)<+\infty$, we have that $\mathcal{H}^{\frac{1}{p}}\left(C_{p, q}\right)=0$. On the other hand, if $q>0$, then $(1 / p, 0)<_{l}(1 / p, q)$ and $\mathcal{H}^{p, q}\left(C_{p, q}\right)>0$ implies that $\mathcal{H}^{\frac{1}{p}}\left(C_{p, q}\right)=+\infty$. Hence, the family $\left\{h_{p, q}\right\}$ provides a more accurate classification than the usual $\left\{g_{s}\right\}$, i.e., it distinguishes more sets; furthermore, the dimension induced by this family can be seen as the set $((0,1) \times \mathbb{R}) \cup\{(0,0)\} \cup\{(1,0)\}$ ordered with $\leq_{l}$, and the set function dim turns out to be the restriction of this order to $(0,1) \times\{0\}$.

\section{ACKNOWLEDGMENTS}

We thank C. Cabrelli and K. Hare for sharing their insight into this problem and giving us access to their unpublished notes [CHM02].

The authors are also grateful to the referee's useful comments.

\section{REFERENCES}

[Bes39] E. Best. A closed dimensionless linear set. Proc. Edinburgh Math. Soc. (2), 6:105-108, 1939. MR0001824(1:302f)

[BT54] A. S. Besicovitch and S. J. Taylor. On the complementary intervals of a linear closed set of zero Lebesgue measure. J. London Math. Soc., 29:449-459, 1954. MR0064849 (16:344d)

[CHM02] Carlos Cabrelli, K. Hare, and Ursula M. Molter. Some counterexamples for Cantor sets. Unpublished Manuscript, Vanderbilt, 2002.

[CHM97] Carlos A. Cabrelli, Kathryn E. Hare, and Ursula M. Molter. Sums of Cantor sets. Ergodic Theory Dynam. Systems, 17(6):1299-1313, 1997. MR1488319 (98k:28009)

[CMMS04] Carlos Cabrelli, Franklin Mendivil, Ursula M. Molter, and Ronald Shonkwiler. On the Hausdorff $h$-measure of Cantor sets. Pacific J. Math., 217(1):45-59, 2004. MR2105765 (2005h:28013)

[CMPS05] C. Cabrelli, U. Molter, V. Paulauskas, and R. Shonkwiler. Hausdorff measure of $p$ Cantor sets. Real Anal. Exchange, 30(2):413-433, 2004/05. MR 2177411|(2006g:28012)

[Fal97] Kenneth Falconer. Techniques in fractal geometry. John Wiley \& Sons Ltd., Chichester, 1997. MR,1449135 (99f:28013) 
[Ols03] L. Olsen. The exact Hausdorff dimension functions of some Cantor sets. Nonlinearity, 16(3):963-970, 2003. MR1975790 (2004g:28009)

[Rog98] C. A. Rogers. Hausdorff measures. Cambridge Mathematical Library. Cambridge University Press, Cambridge, 1998. MR 1692618 (2000b:28009)

[Tri82] Claude Tricot, Jr. Two definitions of fractional dimension. Math. Proc. Cambridge Philos. Soc., 91(1):57-74, 1982. MR633256 (84d:28013)

[Tri95] Claude Tricot. Curves and fractal dimension. Springer-Verlag, New York, 1995. MR.1302173 (95i:28005)

[TT85] S. James Taylor and Claude Tricot. Packing measure, and its evaluation for a Brownian path. Trans. Amer. Math. Soc., 288(2):679-699, 1985. MR776398(87a:28002)

Departamento de Matemática, Facultad de Ingeniería Química, Universidad Nacional del Litoral, Santa Fe, Argentina and IMAL CONICET UNL

E-mail address: igarcia@math.unl.edu.ar

Departamento de Matemática, Facultad de Ciencias Exactas y Naturales, Universidad de Buenos Aires, Ciudad Universitaria, Pabellón I, Capital Federal, Argentina and CONICET, ARgEntina

E-mail address: umolter@dm.uba.ar

Departamento de Matemática, Universidad Nacional del Litoral, Santa Fe, ArGENTINA

E-mail address: scotto@math.unl.edu.ar 